\title{
Adriamycin-Cyclophosphamide (AC) followed by Paclitaxel-Cisplatin (PC). A highly active neoadjuvant regimen in locally advanced breast cancer

\author{
Daniel Mendoza-Posada1, Teresa Vela-Chavez², Enrique Bargallo-Rocha ${ }^{3}$, \\ Nestor Villafañe ${ }^{4}$, Carlos Gamboa-Vignole ${ }^{5}$ and Claudia Arce*1
}

\author{
Address: ${ }^{1}$ Department of Medical Oncology, INCAN México, ${ }^{2}$ Department of Pathology, INCAN México, ${ }^{3}$ Department of Breast Tumors, INCAN \\ México, ${ }^{4}$ Department of Radiology, INCAN Mexico and ${ }^{5}$ Division of Radiation Oncology, INCAN México \\ Email: Claudia Arce* - haydeearce@hotmail.com \\ * Corresponding author
}

from 24th Annual Meeting of the National Cancer Institute of Mexico

Mexico City, Mexico. 14-17 February 2007

Published: 5 February 2007

BMC Cancer 2007, 7(Suppl I):AI doi:I0.I 186/|47|-2407-7-SI-A I

This article is available from: http://www.biomedcentral.com/I47I-2407/7/SI/AI

(C) 2007 Mendoza-Posada et al; licensee BioMed Central Ltd.

\section{Background}

Locally Advanced Breast Cancer patients require neoadjuvant chemotherapy (NACT), surgery and radiation therapy to optimize the chance of a cure. Pathological responses to NACT, are strongly associated with long-term outcome. Cisplatin has shown antitumor activity in breast cancer, shows synergy with paclitaxel and antitumor activity in anthracycline-resistant breast tumors. As NACT, Paclitaxel-Cisplatin (PC) achieves high pathological responses. On the other hand, dose dense chemotherapy has improved free and overall survival in early breast cancer. This phase II trial was set to evaluate the pathological complete response (pathCR) of this regimen based on 4 cycles of adriamycin/cyclophosphamide (AC) followed by weekly (dose dense) PC. Secondary endpoints were to evaluate the safety profile and recurrence-free survival.

\section{Materials and methods}

Patients with histological confirmation of breast cancer in stage IIB-IIIC and aged 18-75 years. Patients also had good performance status [ECOG] score $\leq 2$, adequate hematological, cardiovascular, renal, and hepatic functions. Patients were excluded if they were pregnant or breast feeding or if they had other malignant tumors. Patients received 4 cycles of AC at $60 / 600 \mathrm{mg} / \mathrm{m} 2$ every 21 days followed by weekly PC at $80 / 30 \mathrm{mg} / \mathrm{m} 2$ respectively, at the end of chemotherapy patients underwent surgery.

\section{Results}

A total of 33 patients were included and were evaluable for toxicity and response; the analysis was done in the intention-to-treat. One patient showed paclitaxel hypersensitivity and was removed from the study. Three patients remained inoperable after chemotherapy, hence, received preoperative radiation. Median age was 48 yearsold (26-73), 66.7\% of cases were in stage IIIA and IIIB, tumor median size was $6 \mathrm{~cm}(2.5-16)$, node median size was $3 \mathrm{~cm}(2-16)$, all patients had clinical positive nodes. ER or PgR were positive in 60.6\%, HER2 was positive in $36.4 \%$ (determined by IHC or FISH). The pathCR rate was $30 \%$, the complete nodal response was 52\%. At 18 months of follow-up 2 patients had distant relapse for a $91 \%$ of recurrence free-survival. The regimen was well tolerated with grade III-IV toxicity observed in $6 \%$ of cases.

\section{Conclusion}

The addition of cisplatin to paclitaxel is well tolerated with no significant increase in toxicity. Interestingly, the $30 \%$ of pathCR observed seems superior to other regimens without cisplatin. 